\title{
Perspectives ouvertes \\ par le développement des turbines-pompes \\ d'accumulation
}

\author{
Prospects for the development \\ of accumulation turbine-pump systems
}

\author{
Paul Jarriand \\ Ets NEYRPIC
}

\section{Introduction}

L'accumulation d'énergie hydraulique par pompage connait un développement important, dans le domaine des moyennes chutes et tend à s'orienter depuis quelques années vers des hauteurs de chutes élevées qui présentent l'intérêt de limiter le volume de stockage. Les sites montagneux se prêtent bien à ce genre d'aménagement et certains maîtres d'œuvre envisagent même la réalisation d'installations souterraines, proches des centres de consommation, pour lesquelles seules les possibilités techniques et l'économie du projet détermineront la chute utilisable. On étudie actuellement des aménagements avec des chutes atteignant 1000 à $1500 \mathrm{~m}$, pour des équipements en montagne ou souterrains. Parallèlement, le développement des centrales sous chute moyenne se caractérisera par l'accroissement des puissances installées et de la puissance unitaire des machines qui atteint maintenant $500 \mathrm{MW}$. Cette évolution est illustrée par la figure 1 qui représente un certain nombre d'aménagements en exploitation ou en cours de réalisation.

\section{Etat actuel de la technique}

\section{Turbines-pompes monoétages}

Le développement de ce type de machine sous des chutes de plus en plus élevées s'est activement poursuivi au cours des cinq dernières années. On peut considérer aujourd'hui que l'étape des $500 \mathrm{~m}$ a été largement franchie pour des machines de plus de $250 \mathrm{MW}$. En France, un exemple d'aménagement sous chute élevée est celui de Montézic, équipé de 4 groupes, dont le premier a démarré en avril 1982. La figure 2 représente la coupe de cette machine dont les caractéristiques sont :

$$
\begin{array}{lll}
\text { en turbine } & N=230 \mathrm{MW} & h=419 \mathrm{~m} ; \\
\text { en pompe } & Q=47,25 \mathrm{~m}^{3} / \mathrm{s} & h=410 \mathrm{~m} .
\end{array}
$$

La vitesse de rotation est de $428 \mathrm{tr} / \mathrm{min}$ et le diamètre de roue de $4010 \mathrm{~mm}$.

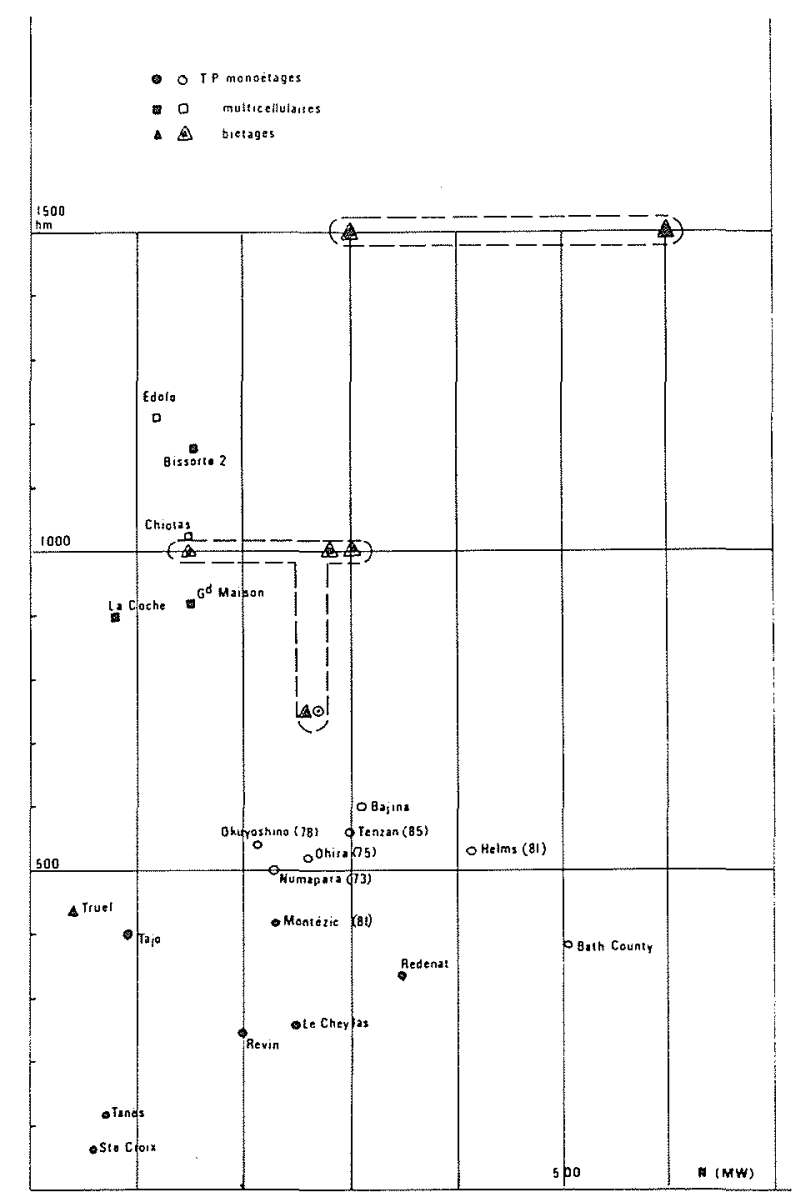

Figure 1 - Evolution des aménagements par pompage en exploit ation ou en cours de réalisation. 


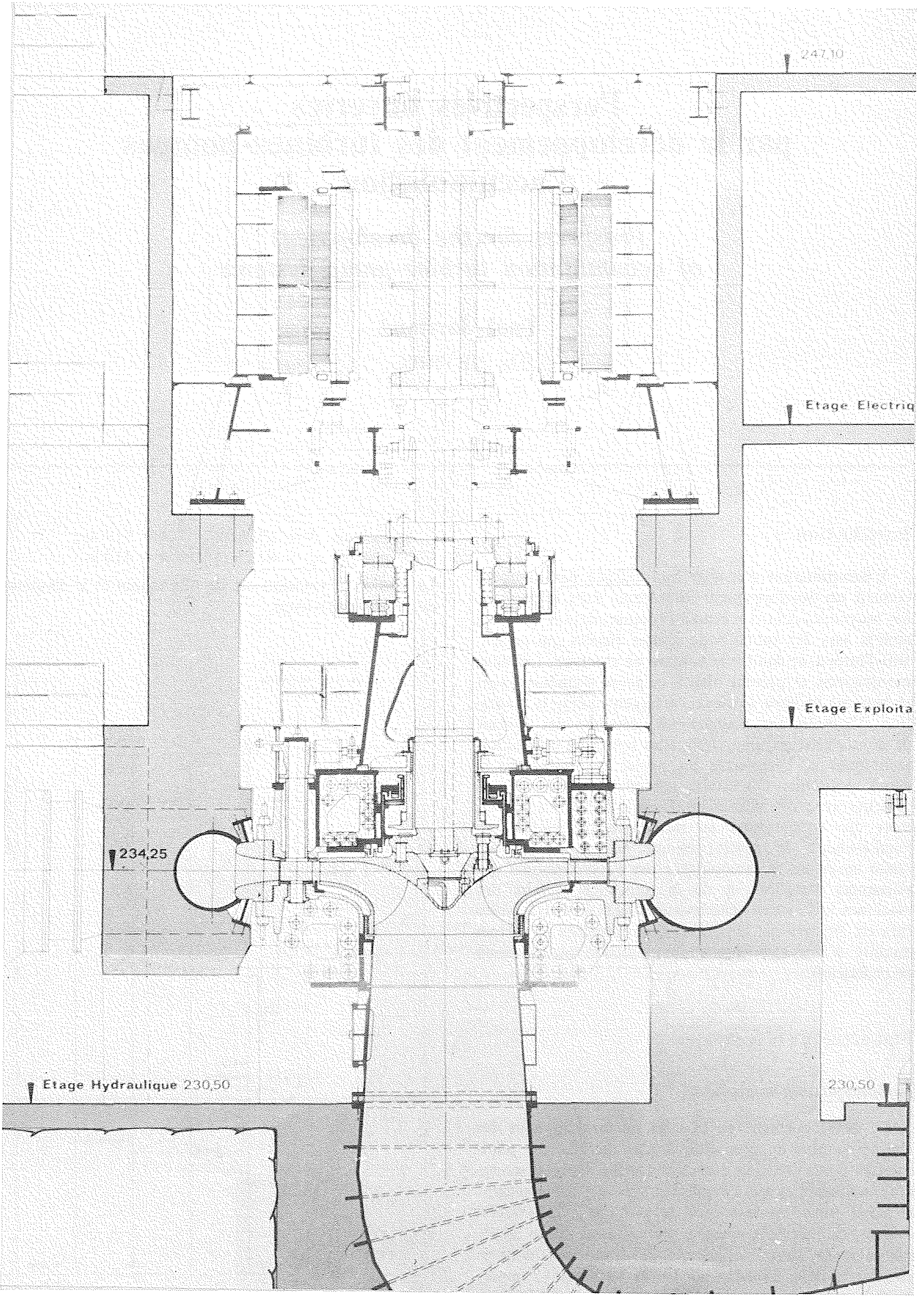

Figure 2 -- Coupe de la turbine-pompe de Montézic. 
Les turbine-pompes monoétages à distributeur mobile permettent un réglage de la puissance entre $40 \%$ et $100 \%$ de la charge maximale avec des conditions de fonctionnement très satisfaisantes. En dessous de $40 \%$, le réglage est évidemment toujours possible, mais des fonctionnements prolongés dans cette zone sont à éviter en raison de l'accroissement sensible des sollicitations dynamiques de certains organes. Le distributeur mobile permet également une variation d'ouverture en pompe en fonction de la hauteur de refoulement, dans le but d'améliorer le rendement surtout dans le cas d'installation ayant un grand marnage. Les prises de charge en pompe et en turbine, ainsi que les passages d'un mode de fonctionnement à un autre, peuvent s'effectuer dans des temps relativement courts répondant ainsi aux exigences des mouvements d'énergie. La possibilité de dénoyer facilement la roue de l'étage unique simplifie les problèmes de démarrage en pompe et autorise le fonctionnement du groupe en compensateur synchrone, ou en réserve tournante.

Dans le cas de Montézic, le démarrage en pompe s'effectue normalement au moyen d'un convertisseur de fréquence, mais il sera également possible de le réaliser en dos à dos avec un machine voisine fonctionnant en turbine.

L'importante campagne de mesures réalisée lors de la mise en service du premier groupe nous a apporté un complément d'informations pour les développements futures de ce type de machine.

\section{Turbines-pompes multicellulaires $\grave{a}$ diffuseurs fixes}

La première installation de ce type est celle de La Coche, en service depuis 1976. Actuellement, sont en fabrication :

- 8 machines à $600 \mathrm{tr} / \mathrm{min}$ sous $971 \mathrm{~m}$ comportant 4 étages pour la centrale de Grand'Maison;

- 4 machines à $600 \mathrm{tr} / \mathrm{min}$ sous $1217 \mathrm{~m}$ comportant 5 étages pour l'usine de Bissorte.

Des centrales de ce type sont en cours de construction ou de mise en service en Italie (Chiotas - Piastra et Edolo) et en Espagne (Moralets).

Les machines de $\mathrm{La}$ Coche, dont la coupe est donnée par la figure 3 , ont comme caractéristiques :

$$
\begin{aligned}
& \text { en turbine } N=\mathrm{MW} \quad h=930 \mathrm{~m} \\
& \text { en pompe } Q=8,50 \mathrm{~m}^{3} / \mathrm{s} \text { sous } h=890 \mathrm{~m}
\end{aligned}
$$

La machine tourne à $600 \mathrm{tr} / \mathrm{min}$ et comporte 5 étages. Sa hauteur de refoulement maximale atteint $944 \mathrm{~m}$.

En l'absence de distributeur mobile, le réglage de la puissance en turbine ne peut être réalisé que par paliers, de valeurs égales à la puissance d'un groupe. L'ouverture des aubes fixes des redresseurs correspond à l'ouverture optimale en pompe, inférieure à l'ouverture de pleine puissance en turbine; il en résulte une perte de puissance de l'ordre de $15 \%$.

Actuellement, le démarrage en pompe de ces machines s'effectue roues noyées. Le couple de barbotage de la pompe à débit nul, qui peut atteindre $70 \%$ du couple nominal, interdit l'emploi des procédés classiques de lancement, tels que moteur Poney ou convertisseur statique. On ne peut envisager pour l'instant que le démarrage asynchrone direct ou le lancement en dos à dos à l'aide d'un groupe voisin fonctionnant en turbine. Des procédures de dénoyage et de renoyage des machines multiétages ont été mises au point sur modèle réduit, mais leur application industrielle reste à développer.

Le démarrage de la turbine et de son couplage, à l'aide de l'organe de garde placé sous le contrôle du régulateur de vitesse, impose que des dispositions soient prises pour assurer la dissipation d'énergie des jets dans les conduits et éviter des vibrations néfastes.

Ces machines ne disposant pas d'organes de réglages sont simples et robustes. Beaucoup plus compactes que les groupes ternaires utilisés jusqu'ici, elles permettent une réduction importante des dimensions de l'usine. Les volumes d'excavation nécessités par l'implantation de ces deux types de groupes sont comparés sur la figure 4. Cette réduction importante des travaux de génie civil, jointe au moindre coût du matériel et à la simplification de l'appareillage de commande-contrôle, conduit à une économie substantielle qui, dans le cas de La Coche, a été estimée par EDF à $12 \%$ du coût total de l'aménagement.

\section{Turbines-pompes biétages à distributeurs réglables}

Lorsque le réglage de la puissance des groupes de hautes chutes s'impose, il devient nécessaire d'utiliser des distributeurs réglables. La turbine-pompe biétage, à double réglage, semble la solution la plus intéressante actuellement. Sous l'impulsion d'EDF, Neyrpic a été amené à étudier ce type de machine et le groupe expérimental du Truel, qui sera mis en service fin de l'année 1982, constituera très probablement une première réalisation mondiale. La figure 5 représente la coupe de cette machine dont les caractéristiques sont les suivantes :

puissance en turbine $N=38 \mathrm{MW} \quad$ sous $h=438 \mathrm{~m}$ débit en turbine $\quad Q_{T}=10,2 \mathrm{~m}^{3} / \mathrm{s}$

débit en pompe $\quad Q_{P}=6,9 \mathrm{~m}^{3} / \mathrm{s}$

vitesse de rotation $\quad n=750 \mathrm{tr} / \mathrm{min}$

diamètre de roue $\quad D=1675 \mathrm{~mm}$

Cette machine a été réalisée sans rechercher l'optimisation des formes hydrauliques, la préoccupation essentielle étant de construire une unité largement dimentionnée, permettant d'effectuer sans risque tous les essais souhaitables. Les essais prévus sur ce groupe expérimental permettront une analyse approfondie $\mathrm{du}$ comportement de la turbine-pompe non seulement durant les régimes normaux d'exploitation, mais également en fonctionnements transitoires et dans certaines conditions exceptionnelles, qui ne sont actuellement étudiées que sur modèle réduit. En particulier, l'entraînement en dos à dos de cette machine par une Pelton de la centrale voisine du Pouget autorisera des fonctionnements en régime permanent dans les quadrants "pompe-frein" et "pompe à rotation inversée". Des dispositions sont prises pour étudier l'influence d'une désynchronisation des distributeurs. Les séquences de dénoyage et de renoyage des roues lors des démarrages en pompe ou des passages de turbine à compensateur pourront être mises au point. 


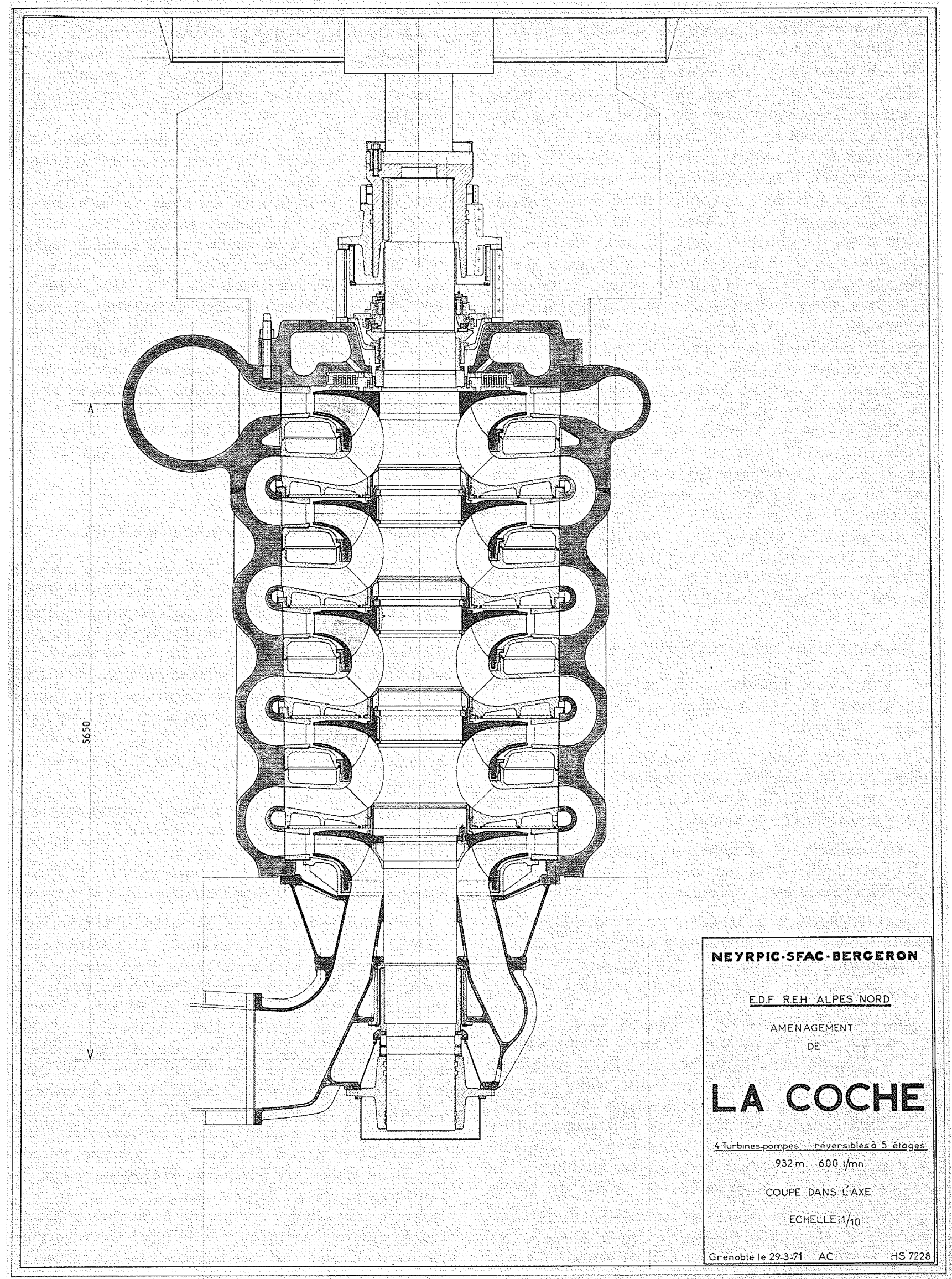

Figure 3 - Coupe de la turbine-pompe de La Coche. 


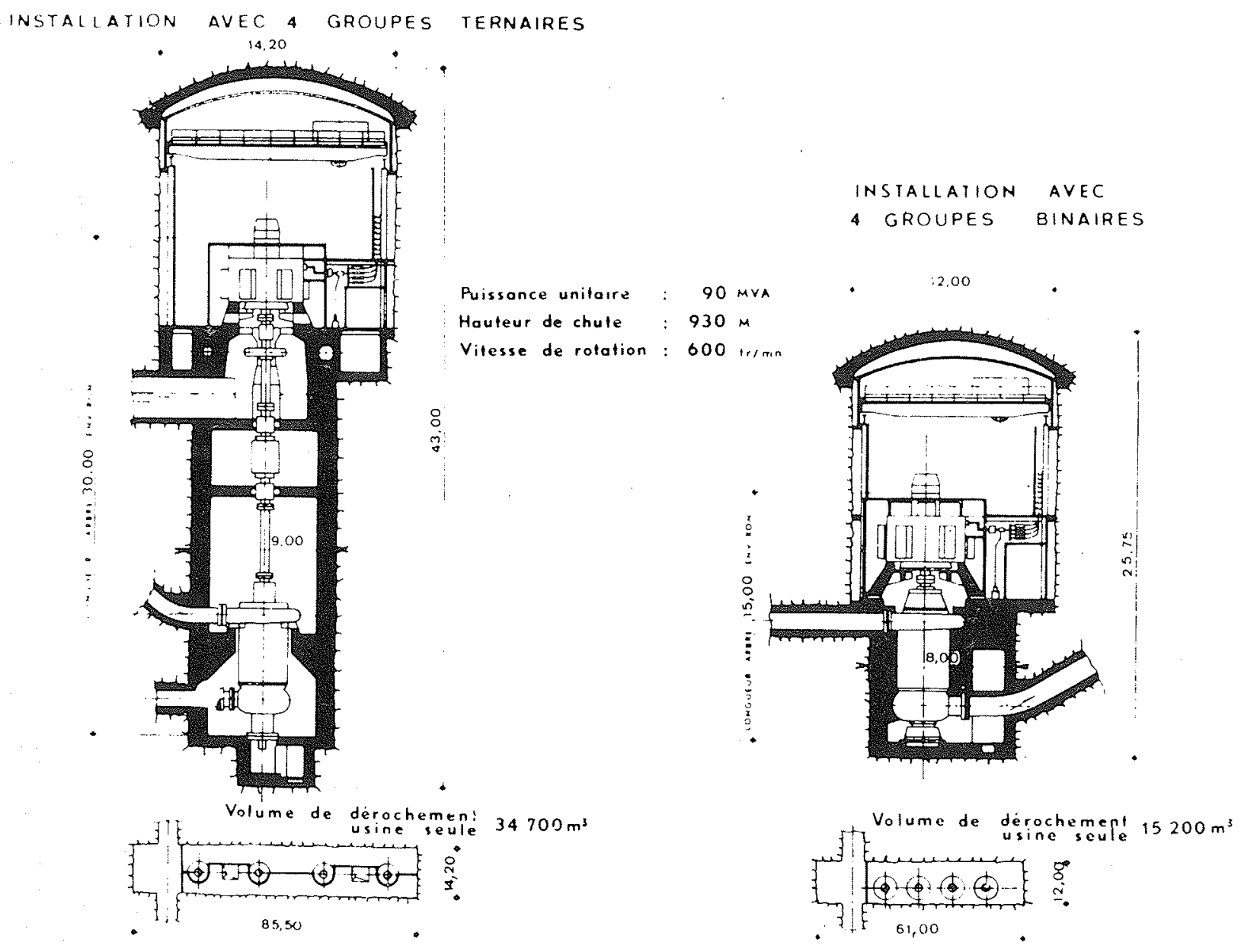

Figure 4 - Comparaison des volumes d'excavation nécessités par l'implantation de deux types de groupe.

Les informations tirées de ces essais constitueront une aide précieuse au développement de ces turbinespompes qui présenteront, par rapport aux multicellulaires, les mêmes avantages que les machines monoétages; à savoir :

- gain de puissance en turbine,

- possibilité de modulation de la puissance ;

- facilité de démarrage en pompe, roues dénoyées et distributeurs fermés, le couple résistant au synchronisme étant alors inférieur à $10 \%$ du couple nominal ;

- possibilité de fonctionnement en compensateur synchrone.

Problèmes posés par la conception et la réalisation des turbines-pompes de haute chute

L'extension du domaine d'exploitation des turbinespompes vers les très hautes chutes pose des problèmes difficiles, tant hydrauliques que mécaniques, dont nous examinerons les principaux aspects.

\section{Performances hydrauliques}

\section{Cavitation en pompe}

C'est le fonctionnement en pompe qui conditionne le problème de cavitation des turbines-pompes.

La figure 6 montre la répartition des pressions du côté déprimé d'un même profil équipant respectivement une turbine et une pompe.

On constate l'importance de la dépression à l'entrée de l'aube qui joue, pour le fonctionnement en pompe, un rôle dominant alors que pour la turbine, cette dépression est masquée par la valeur de la pression moyenne, encore importante à cet endroit. Cette pointe de dépression, génératrice de cavitation pour la pompe, est évidemment d'autant plus importante que la chute est plus élevée.

Pour les très hautes chutes, deux aspects particulièrement délicats concernent la cavitation :

- le premier est lié à la grande sensibilité du phénomène relativement à la forme de l'arête d'entrée, à l'ouie ;

- le deuxième est lié à l'intensité d'usure qui est fortement influencée par la vitesse d'écoulement. 


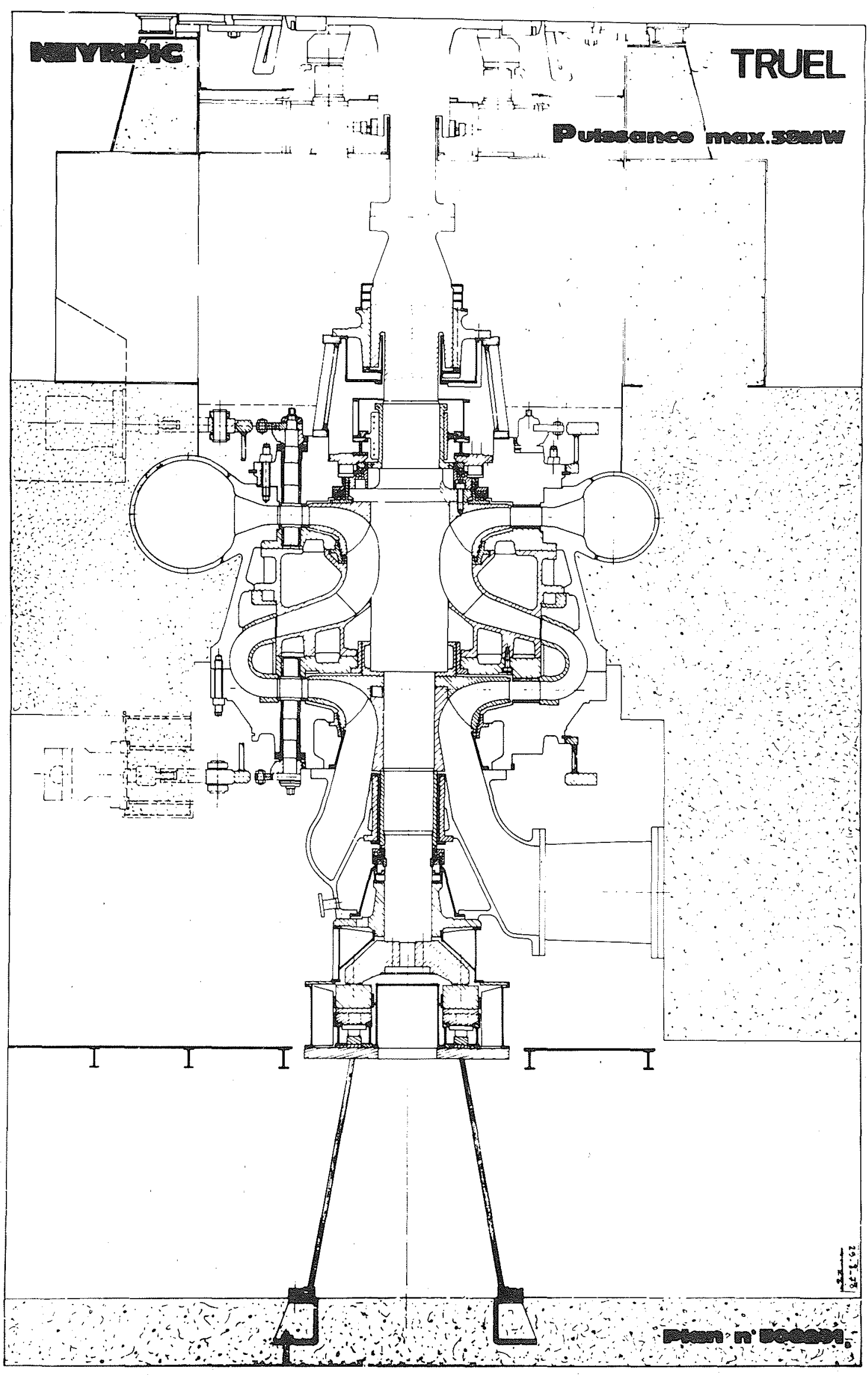

Figure 5 - Coupe de la turbine-pompe bi-étage du Truel. 
L'influence de la forme du bord d'attaque des profils sur le niveau du minimum de la courbe de pression est mise en évidence sur la figure 7 . On y remarque le résultat d'un écart de forme du bord d'entrée, relativement faible, mais qui conduirait pour une machine fonctionnant sous $600 \mathrm{~m}$ à une différence de calage de $7 \mathrm{~m}$ environ. La précision de réalisation des formes d'aubage revêt donc une importance fondamentale.

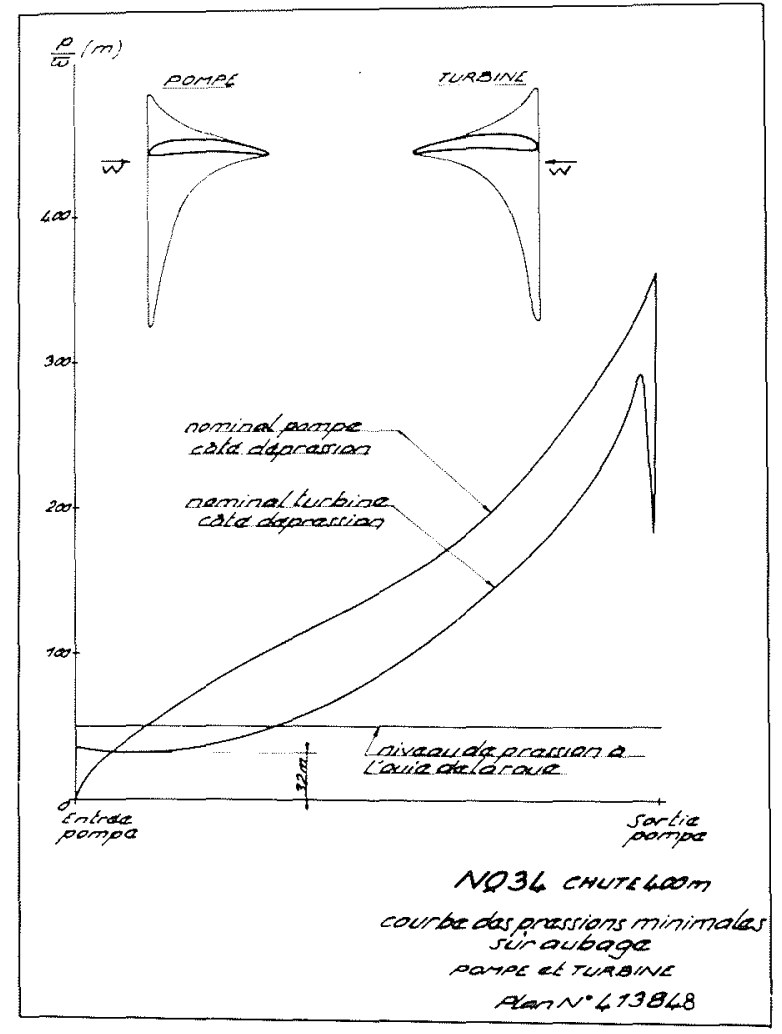

Figure 6 - Répartition des pressions, côté déprimé, d'un même profil équipant :

a) - une turbine,

b) - une pompe

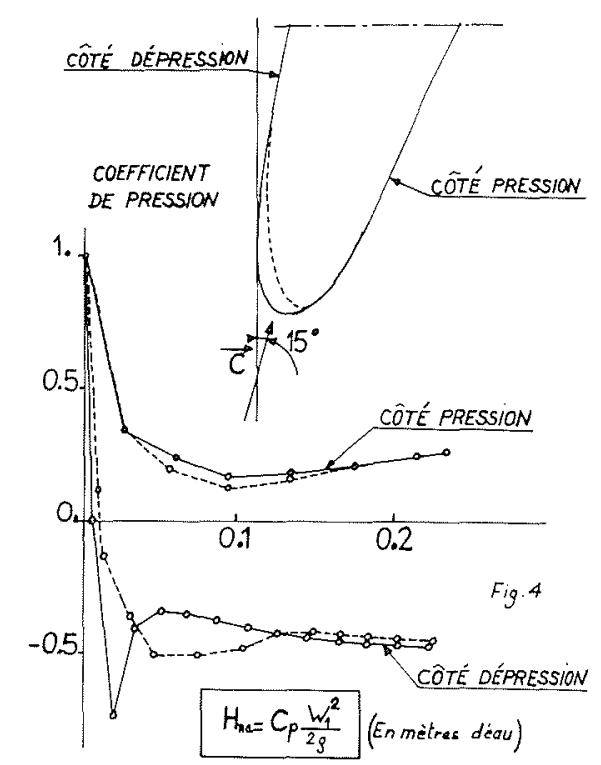

Figure 7 - Influence de la forme du bord d'attaque des profils sur le niveau du minimum de la courbe de pression.

\section{Choix de la vitesse spécifique}

Pour les turbines-pompes monoétages, l'optimisation globale d'un projet sous une chute donnée conduit à une valeur de $n_{q}$ qui caractérise le tracé hydraulique. Cette optimisation continuellement améliorée par l'expérience et le progrès des recherches montre que le $n_{q}$ décroit lorsque la chute augmente. La figure 8 donne l'évolution générale des vitesses spécifiques des turbinespompes en fonction de la hauteur de refoulement. Pour les très hautes chutes, il paraît logique d'envisager l'extrapolation de la courbe. Par exemple, pour une chute de $800 \mathrm{~m}$, il semblerait que l'on puisse utiliser une roue de vitesse spécifique inférieure à 25. Mais ce type de machine, très fortement centrifuge, présente quelques inconvénients :

- pertes par frottements "disques" très importantes (voir fig. 9 donnant les rendements maximaux en fonction de la vitesse spécifique) ;

- difficultés de réalisation des roues, pour lesquelles la trop faible hauteur interdit presque tout accès pour le contrôle ou le meulage.

Si l'on essaye d'augmenter cette vitesse spécifique

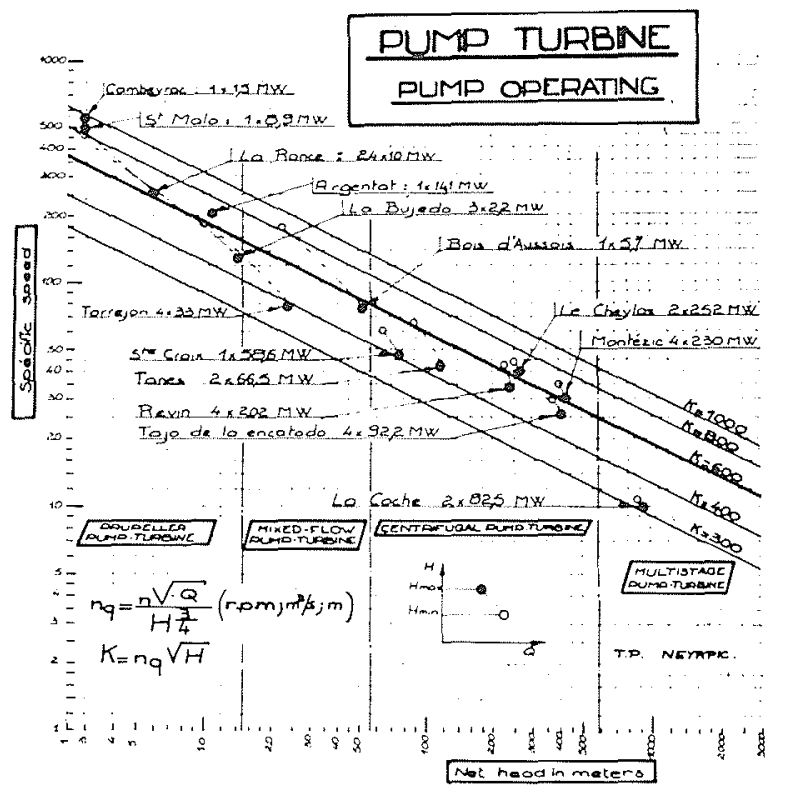

Figure 8 - Evolution générale des vitesses spécifiques des turbines-pompes en fonction de la hauteur de refoulement.

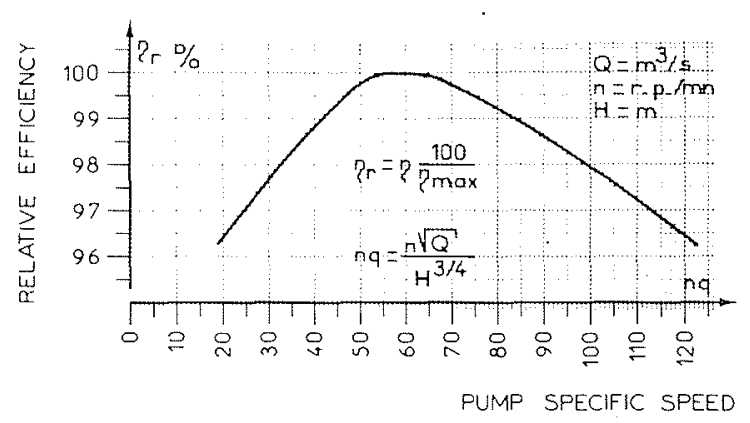

Figure 9 - Rendement maximal d'une turbine-pompe en fonction de sa vitesse spécifique. 
pour améliorer les rendements et faciliter la construction, nous nous heurtons à d'autres difficultés :

- vitesses d'écoulement trop importantes dans les conduits roue et volute;

- vitesses de rotation de la machine trop grandes par rapport à la puissance, les constructeurs électriciens risquant de ne pouvoir réfrigérer facilement leurs alternateurs,

- diminution du $M D^{2}$ du rotor.

Le même phénomène se produit pour les turbinespompes biétages et s'il est difficile actuellement de tracer pour ce type de machine une courbe de $n_{q}$ limite en fonction de la chute par étage, il est logique d'envisager qu'elle aura la même allure que celle des monoétages. L.influence d'un arbre tournant à l'entrée de la roue du premier étage sur les performances et les problèmes de cavitation impose de choisir des valeurs de $n_{q}$ un peu inférieures à celles de la figure. Pour ces machines, nous retenons des valeurs minimales du $n_{q}$ comprises entre 26 et 37 pour des chutes par étage variant de 750 à $400 \mathrm{~m}$

\section{Conception mécanique}

Lors de la conception et du dimensionnement des turbines-pompes de très haute chute, le constructeur est confronté à des problèmes de types différents selon l'organe de machine considéré. On peut distinguer, en effet, deux modes de sollicitation, caractérisés essentiellement par la nature des forces mises en jeu :

- les charges statiques qui s'appliquent aux parties fixes du groupe ;

- Les charges dynamiques qui agissent surtout sur les organes de vannage, les aubages de la roue, la ligne d'arbre et les paliers.

Une des différences fondamentales entre les turbinespompes et les turbines Francis réside dans le fait que les cycles de charge sont beaucoup plus fréquemment variables pour les premières que les secondes, en raison de leur mode d'exploitation en production de pointe. Plusieurs fois par jour, les machines démarrent et s'arrêtent avec chaque fois un cycle de charge et de décharge et des fonctionnements transitoires. Ceci conduit à des sollicitations très sévères en fatigue.

Il est alors très important de déterminer les forces hydrodynamiques pulsatoires qui peuvent soumettre les organes de la machine à des cycles de contraintes alternées de fréquences souvent voisines de certaines des fréquences propres des structures composantes.

Pour les turbines pompes biétages, le fractionnement de la chute et une vitesse de rotation plus faible conduisent à une diminution des sollicitations dynamiques sur les directrices et les roues.

L'avant-distributeur est la pièce vitale de la machine, car c'est lui qui supporte tous les efforts de la turbinepompe, qu'ils viennent de la volute, de la pression de l'eau sur ses propres surfaces, ou des boulonnages de fixation des flasques. Les charges transmises par ces derniers peuvent dépasser $20000 \mathrm{t}$ pour des turbinespompes biétages de $300 \mathrm{MW}$ sous $1000 \mathrm{~m}$.

Les flasques supérieur et inférieur doivent présenter le maximum de rigidité et leur étude, par la méthode des éléments finis, permet d'abaisser le niveau des contraintes en agissant sur les différents paramètres.

Les directrices d'une turbine-pompe sont soumises, au cours des fonctionnements transitoires et notamment au cours d'un emballement, à des couples pulsatoires importants. Leur importance dépend de la valeur de la fréquence propre de torsion de la directrice comparée à la fréquence de défilement des aubes de la roue et à son premier harmonique.

Nous nous imposons que la première fréquence propre dans l'eau soit comprise entre 2 et 3 fois la fréquence d'excitation à la vitesse de rotation transitoire maximale.

L'analyse des contraintes statiques et dynamiques des roues est effectuée par la méthode des éléments finis. La détermination des épaisseurs du plafond, de la ceinture et des aubes doit être faite avec grand soin pour éviter les fissurations en service, notamment aux bords de sortie des aubes.

Les charges statiques et dynamiques croissent avec la chute et la vitesse de rotation; l'augmentation corrélative de l'épaisseur des directrices et des aubes de la roue rendent plus difficile le tracé hydraulique de la machine.

\section{Régimes transitoires}

Les sollicitations maximales des divers organes des turbines-pompes apparaissent durant les régimes transitoires, en particulier dans le quadrant pompe-frein, à l'emballement en turbine et en pompe-frein à rotation inversée (voir Fig. 10). L'amplitude des fluctuations dépend du cheminement du point figuratif dans ces différentes zones; celui-ci est étroitement lié aux caractéristiques des adductions et au $M D^{2}$ de l'alternateur qui imposent une loi de fermeture du vannage plus ou moins favorable.

Lorsque l'aménagement comporte une conduite unique et une évacuation commune à plusieurs groupes, des surpressions ou dépressions excessives peuvent être induites par l'interaction des machines, du fait de la forme en " $S$ " de la caractéristique de la turbine. Pour les turbines-pompes de très haute chute, il importe que le système des adductions soit harmonisé avec le groupe dès le début du projet.

\section{Perspectives d'avenir}

\section{Projets français}

Le programme hydraulique d'EDF, en machines réversibles, envisage l'aménagement de divers sites dont les domaines de chutes se situeront entre 750 et $1500 \mathrm{~m}$. Au stade actuel, nos études de faisabilité, limitées à des chutes de $750 \mathrm{~m}$ et $1000 \mathrm{~m}$, ont été entreprises sur des exemples d'aménagement fournis par les régions d'équipement.

\section{Chutes de $750 \mathrm{~m}$}

Pour cette chute, les deux solutions mono et biétage sont envisageables. Toutefois, dans la mesure où elle 


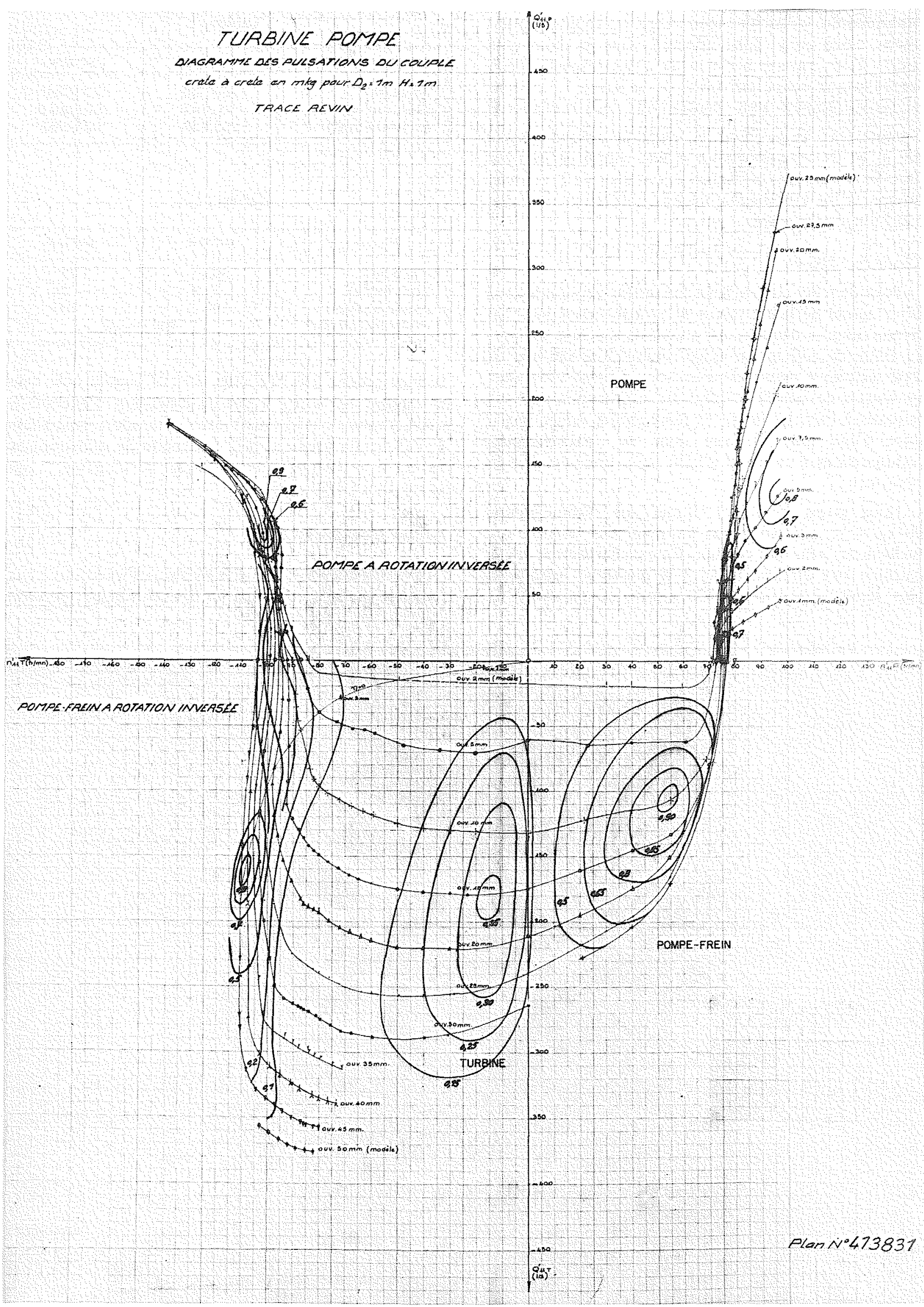

Figure 10 - 
est réalisable, la machine monoétage sera toujours moins chère. Nous avons donc effectué, dans le cadre d'un projet EDF, l'étude préliminaire d'une machine représentée par la coupe provisoire de la figure 11 et dont les caractéristiques sont mentionnées dans le tableau I.

Compte tenu de la chute, la vitesse spécifique retenue est $n_{q}=26$ ce qui conduit à une vitesse de rotation de $600 \mathrm{tr} / \mathrm{mn}$. Les prétudes mécaniques de la turbinepompe et de l'alternateur-moteur confirment la faisabilité d'une telle unité.

\section{Chute de $1000 \mathrm{~m}$}

Pour de telles chutes, la seule solution applicable dans l'état actuel de la technique et probablement pour un certain nombre d'années est encore la machine biétage. La chute de $500 \mathrm{~m}$ par étage conduit à retenir une vitesse spécifique maximale d'environ 31 pour rester dans les limites de constructibilité des alternateurs moteurs refroidis à l'air. La figure 12 représente la coupe provisoire d'une telle machine dont les caractéristiques correspondant à un projet d'EDF sont précisées dans le tableau II.

Nous avons également étudié une turbine-pompe de $n_{q}=26$ correspondant au minimum possible actuellement, afin de préciser les caractéristiques de la machine aux dimensions et à la puissance unitaire la plus faible. Tournant à $750 \mathrm{tr} / \mathrm{mn}$, sa puissance en
Tableau I

\begin{tabular}{|l|c|c|}
\hline & turbine & pompe \\
\cline { 2 - 3 } chute $(\mathrm{m})$ & $765 / 706$ & $773 / 718$ \\
débit $\left(\mathrm{m}^{3} / \mathrm{s}\right)$ & $45,3 / 43$ & $31,6 / 36,9$ \\
puissance (MW) & $298 / 162$ & $271 / 292$ \\
\hline
\end{tabular}

Tableau II

\begin{tabular}{|l|c|r|}
\hline & turbine & pompe \\
\cline { 2 - 3 } chute $(\mathrm{m})$ & 993 & 1011 \\
débit $\left(\mathrm{m}^{3} / \mathrm{s}\right)$ & 34,9 & 23 \\
puissance $(\mathrm{MW})$ & 296 & 264 \\
vitesse de rotation & \multicolumn{2}{|c|}{$600 \mathrm{tr} / \mathrm{min}$} \\
\hline
\end{tabular}

turbine atteint $135 \mathrm{MW}$ (Fig. 15). Il semble que, dans la mesure où il n'y a pas de limitation d'encombrement imposée par les conditions de transport, la solution à $600 \mathrm{tr} / \mathrm{min}$ présente plus de facilité de dimensionnement, de réalisation, d'accessibilité, de montage... que la solution à $750 \mathrm{tr} / \mathrm{min}$.

\section{Projets étrangers}

Les Ingénieurs Conseils américains s'intéressent aux turbines pompes, à simple ou double réglage, pouvant

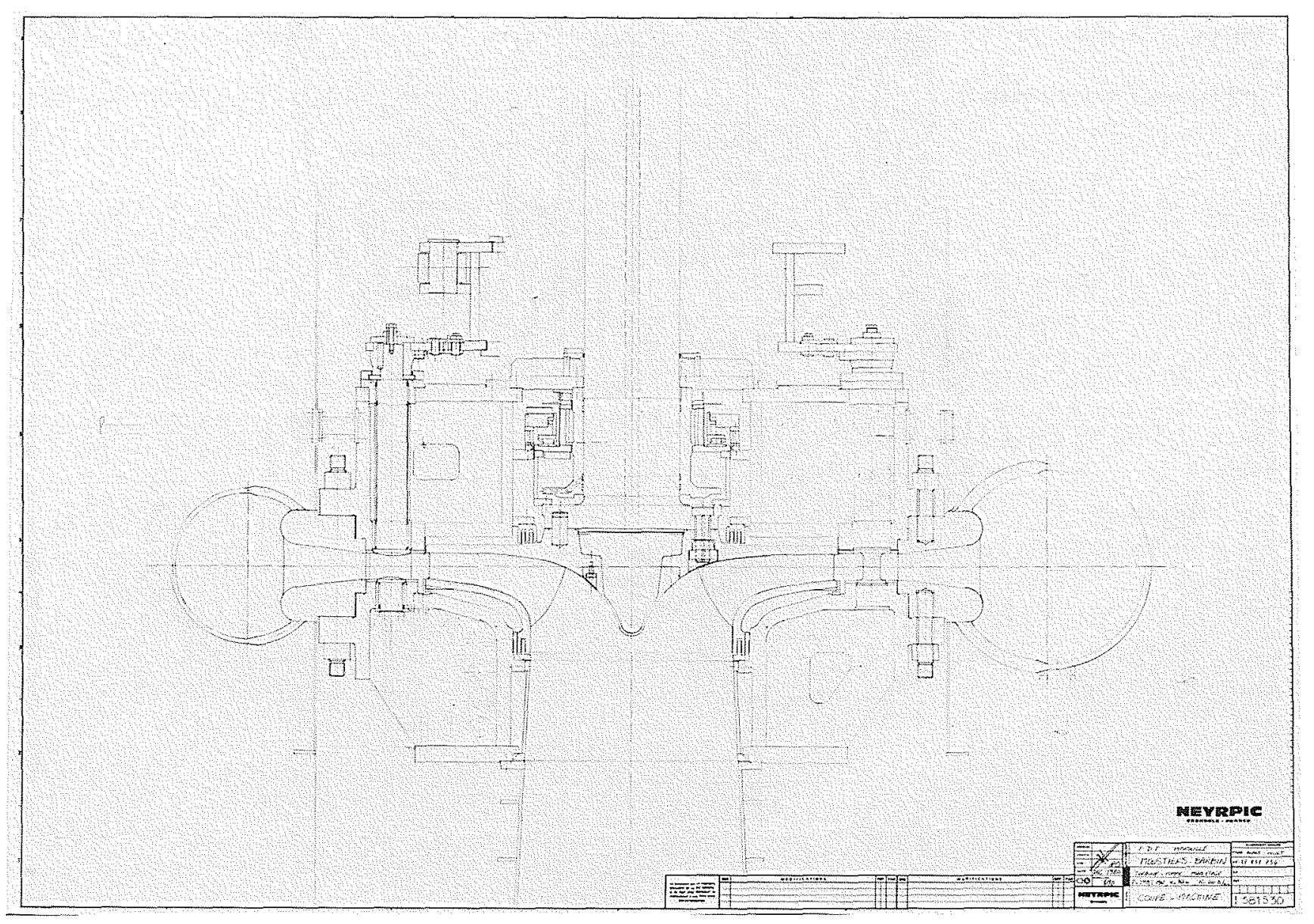

Figure 11 - Coupe provisioire d'une turbine-pompe mono-étage pour chute de 750 mètres tournant à une vitesse de $600 \mathrm{tr} / \mathrm{min}$. 


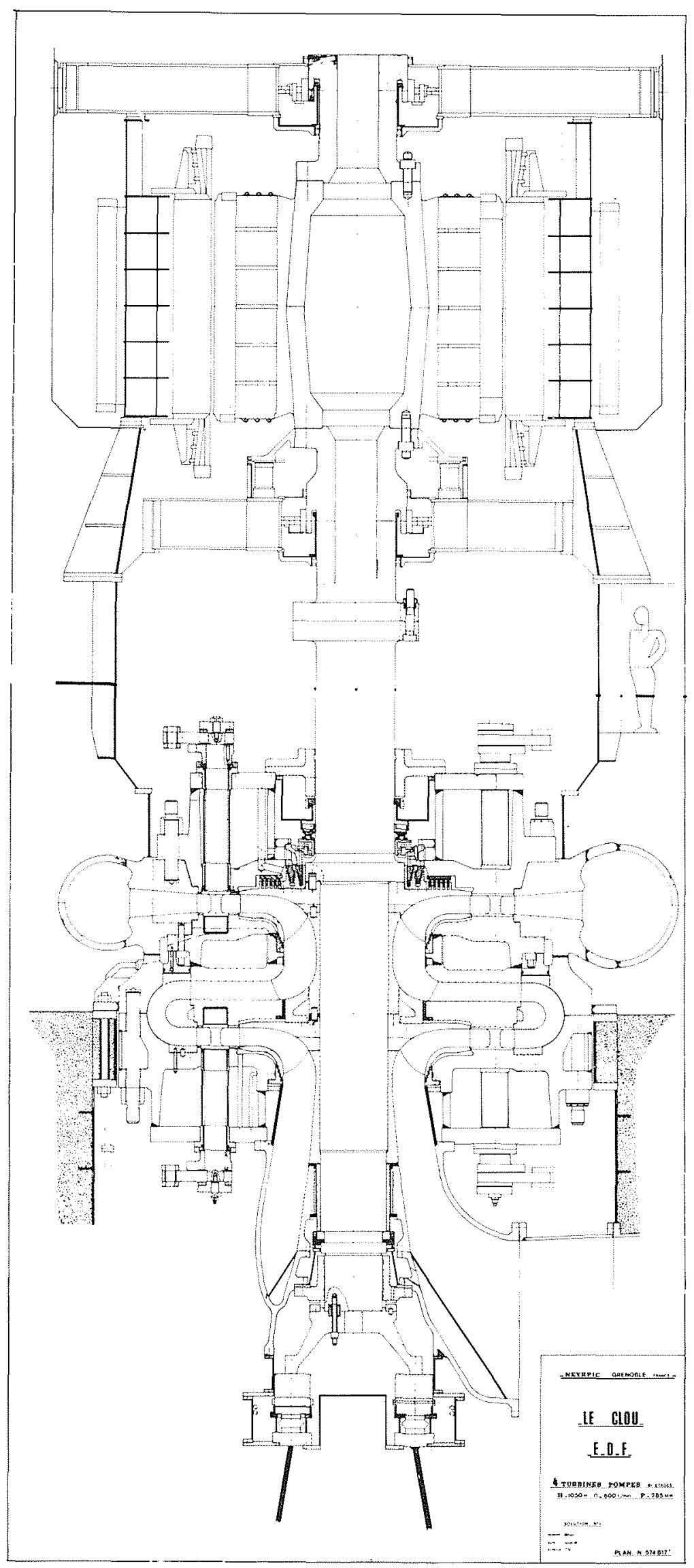

Figure 12 - Coupe provisoire d'une turbine-pompe bi-étage pour chute de 1000 mètres tournant à une vitesse de $600 \mathrm{tr} / \mathrm{min}$. 


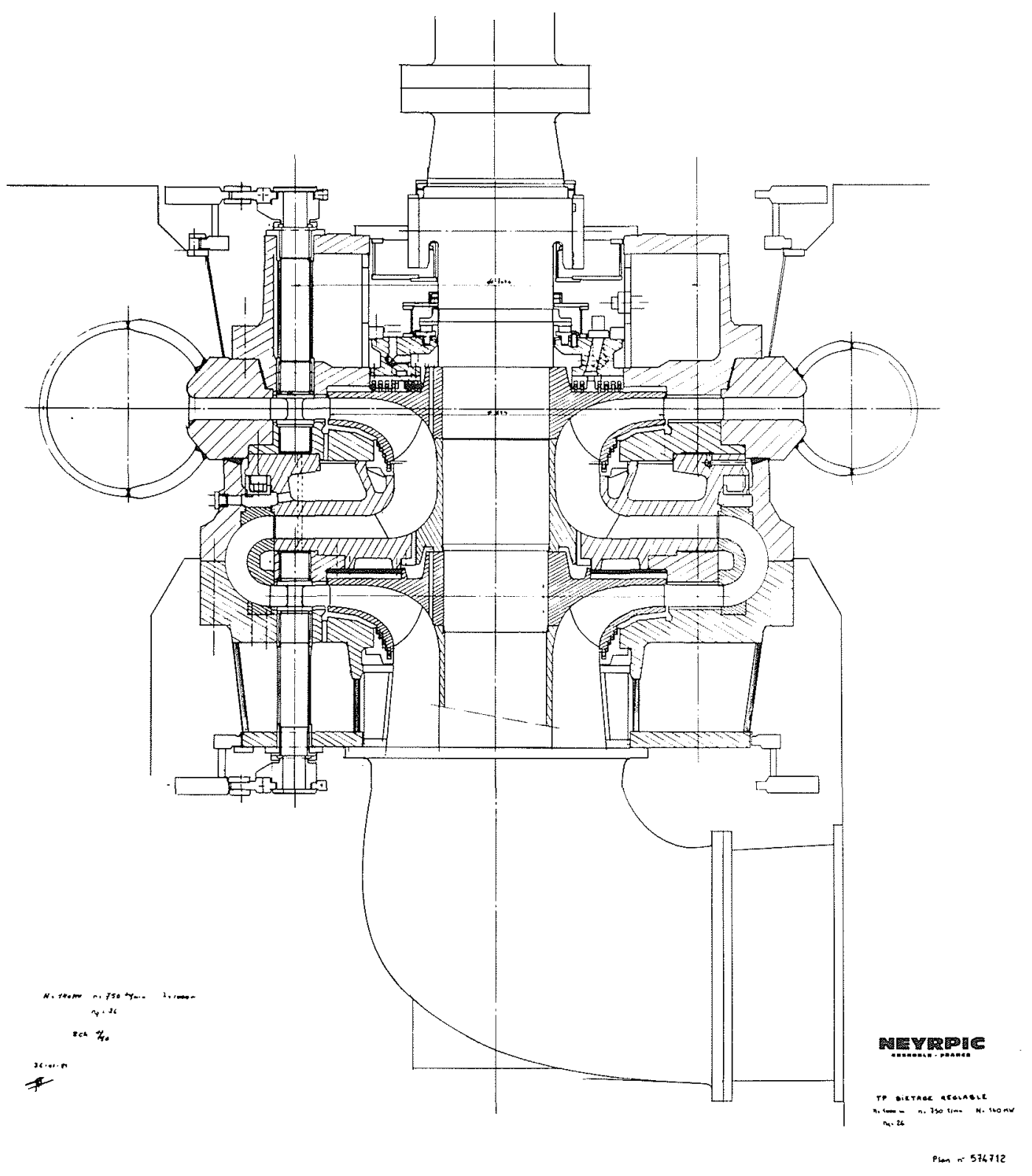

Figure 13 - Coupe provisoire d'une turbine-pompe biétage pour chute de 1000 mètres tournant à la vitesse de $750 \mathrm{tr} / \mathrm{min}$.

développer des puissances unitaires de 300 à $600 \mathrm{MW}$ sous $1500 \mathrm{~m}$ de chute. Nous avons été récemment consultés pour la préparation d'un contrat d'étude sur ce type d'installation.

\section{Conclusions}

Les turbines-pompes de hautes chutes connaîtront vraisemblablement un développement important dans les années à venir. Le développement des installations sous chutes moyennes se poursuivra avec des machines monoétages. Pour les hautes chutes, trois types fondamentaux pourront être envisagés :

- les turbines-pompes monoétages à distributeur mobile - les turbines-pompes multicellulaires à diffuseurs fixes - les turbines-pompes biétages à distributeurs mobiles.
Les machines multicellulaires pourront être envisagées pour des chutes atteignant $2000 \mathrm{~m}$ sous réserve que les problèmes de démarrage soient convenablement résolus et que l'on puisse s'accommoder de l'absence de réglage de la puissance en turbine. Les impératifs des mouvements d'énergie demanderont aux futures centrales d'accumulation qu'elles assurent des démarrages, des prises de charge, des transferts d'un mode de fonctionnement à un autre, en des temps relativement courts. D'autre part, la modulation de la puissance en turbine qui sera affectée d'une valorisation toujours plus élevée, ajoute encore de l'intérêt au développement des machines de hautes chutes et de grande puissance à distributeurs mobiles.

On peut considérer actuellement que le domaine d'emploi des turbines pompes monoétages peut s'étendre jusqu'à des chutes d'environ $800 \mathrm{~m}$. Enfin, 
le développement des machines biétages pour des chutes atteignant 1000 à $1500 \mathrm{~m}$ devra suivre celui des turbines-pompes mono-étages équipant des chutes de 500 à $750 \mathrm{~m}$. Les travaux que nous entreprenons montrent que cette évolution pourrait se concrétiser à moyen terme, du moins pour des chutes allant jusqu'à $1100 \mathrm{~m}$.
Au-delà, des études très poussées seront indispensables, tant dans les domaines hydrauliques et mécaniques des turbo-machines que dans celui des alternateurs moteurs, dont les problèmes actuels de refroidissement devront être résolus pour permettre l'accroissement des puissances unitaires. 